$$
A D-A 228646
$$

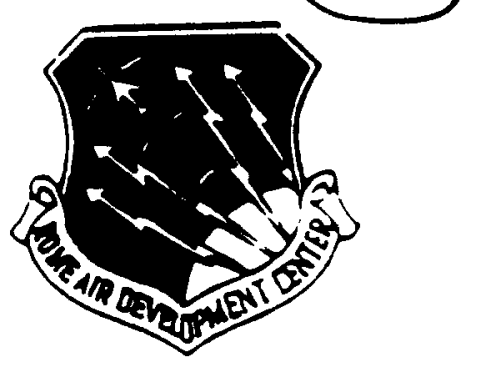

\title{
ERROR BOUNDS AND ASYMPTOTIC PERFORMANCE UNDER MISMATCH OF MULTISENSOR DETECTION SYSTEMS
}

Syracuse University

D. Kazakos

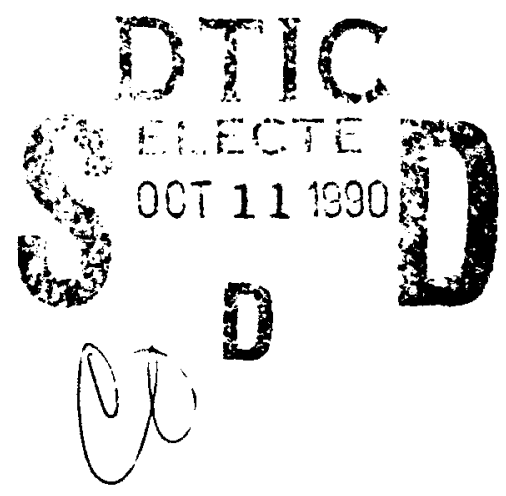

ASPROVED FOR PUBLIC RELEASE; DISTRIBUTION UNLIMITED.

Rome Air Development Center Air Force Systems Command Griffiss Ali Force Basf, NY 13441-5700 


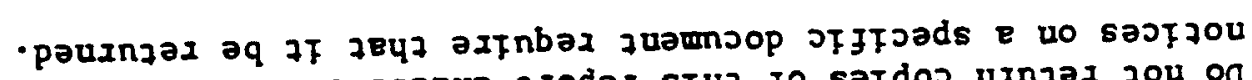

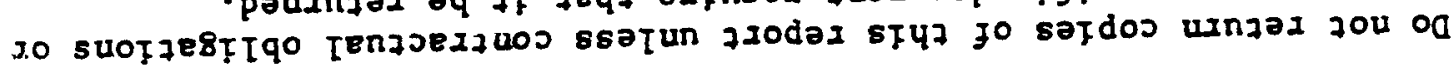

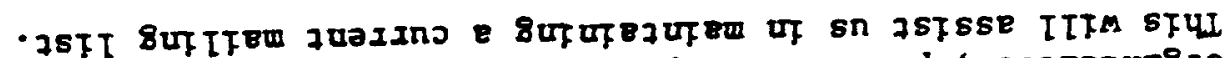

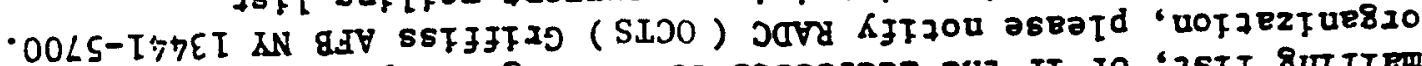

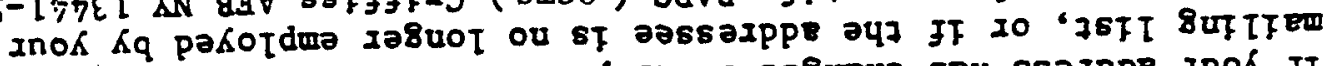

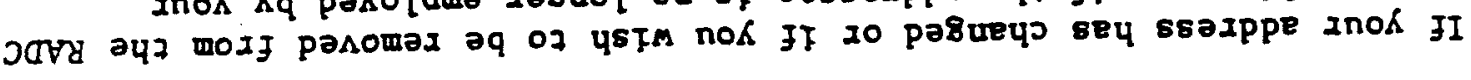

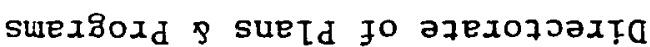

$\mathrm{ZIIZ} \cdot \mathrm{V}$ NHOS

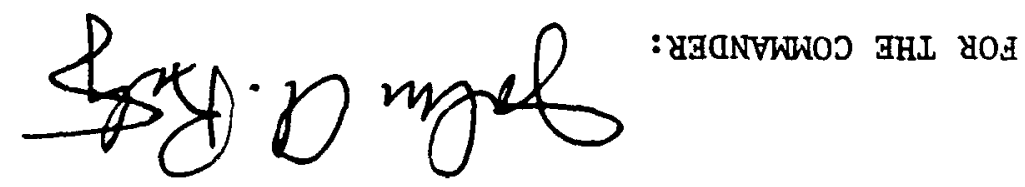

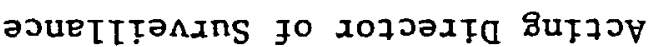

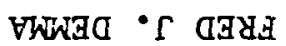

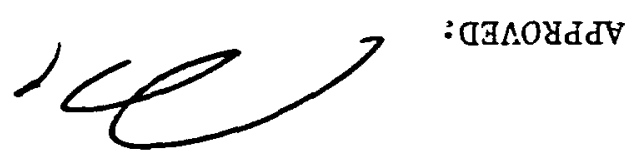

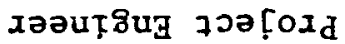

VTOOINNVA 0 INGONIA

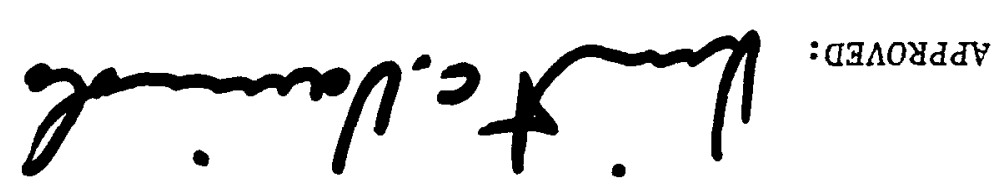

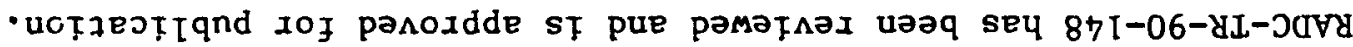

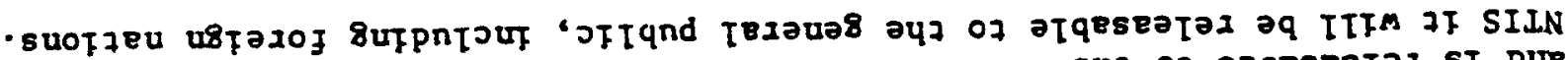

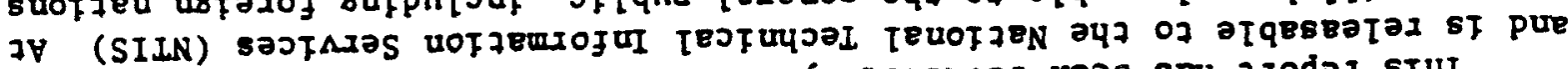

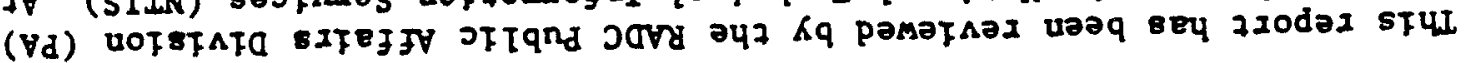




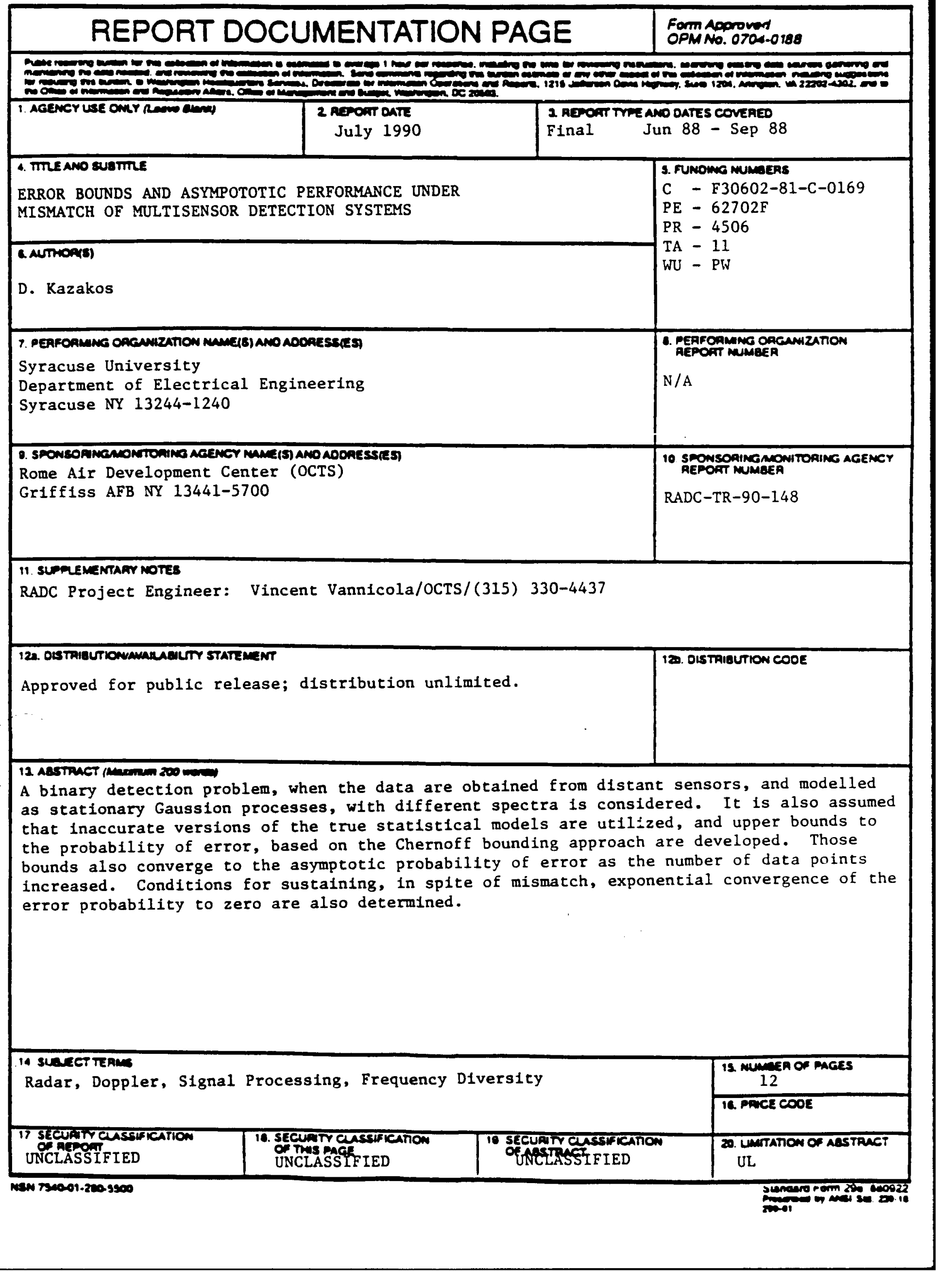




\title{
ERROR BOUNDS AND ASYMPTOTIC PERFORMANCE LNDER MISMATCH OF MULTISENSOR DETECTION SYSTEMS
}

\author{
D. Kazakos \\ Electrical Engineering Department \\ University of Virginia \\ Charlottesville, VA 22901
}

\begin{abstract}
We consider a binary detection problem, when the data are obtained from $m$ distant sensors, and modelled as stationary Gaussion processes, with different spectra. We also assume that inaccurate versions of the true statistical models are utilized, and we develop upper bounds to the probability of error, based on the Chernotf bounding approach. Those bounds also converge to the asymptotic probability of error as the number $\mathbf{n}$ of data points increases to $\infty$. Conditions for sustaining, in spite of mismatch, exponential convergence of the error probability to zero with $n$ are determined.
\end{abstract}

\section{INTRODUCTION}

There has been much interest recently in the signal detection problem for data available from multiple sensors [1] - [7]. In this paper we develop bounds to the probability of error for binary detection from multisensor data. when inaccurate versions of the actual statistics are incorporated into the decision rule. We specifically investigate the error probability for detection in Gaussian, stationary processes with inaccurately known spectra.

\section{SUMIMARY OF RESULTS}

Suppose that $m$ sensors are urilized for deciding between two hypotheses, $H_{1}, H_{0}$. Let $x_{k}=\left(x_{k 1} \cdots x_{k \pi}\right)$ be the data vector for the $k$ th sensor, distributed according to the probability density function $f_{j k}\left(x_{k}^{n}\right)$ for $j=0.1$ under $H_{h}, H_{1}$, correspondingly. Suppose. also, that $g_{j k}\left(x_{k}^{n}\right)$ is the "inaccurate" version of $f_{k}\left(x_{k}^{n}\right)$ that is used in the decision rule. We will assume throughout this paper that the data from different sensors are statistically independent. This assumprion will be removed in a subsequent paper. Because of the independence between distinct sensors, the likelihood ratio test is:

$$
\text { Decide } H_{1} \text { if } m^{-1} \cdot \sum_{k=1}^{m} q_{k}\left(x_{k}^{n}\right)>T
$$

where:

$$
\Psi_{k}\left(x_{k}^{\beta}\right)=n^{-1} \cdot \log \frac{g_{i k}\left(x_{k}^{n}\right)}{g_{0 k}\left(x_{k}^{n}\right)}
$$

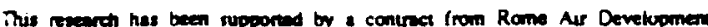

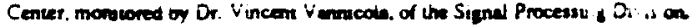

is the "mismatched" log likelihood function of the kth sensor.

Let, for $\mathrm{j}=0,1$ :

$P_{j}(f, g, n)=$ Probability of erroneously deciding $H_{j}$ using $\left\{\mathrm{g}_{\mathbf{j k}}\right\}$ and based on $\mathrm{n}$ measurements.

We will utilize the Chemotf bounding technique. and subsequentiy relate the bound to "large deviation theory." $[8],[9]$, as $n \rightarrow \infty$.

The basic Chemoff bound is:

$$
\begin{aligned}
P_{0}(f, g, n)= & \operatorname{Pr}\left[m^{-1} \sum_{k=1}^{m} z_{k}>T \mid H_{0}\right] \\
& \leq E_{o} \exp \left[t\left(m^{-1} \sum_{k=1}^{m} z_{k}-T\right)\right]= \\
= & \prod_{k=1}^{m} E_{o} \exp \left[\mathrm{tm}^{-1}\left(z_{k}-T\right)\right] \\
P_{1}(f, g, n)= & \operatorname{Pr}\left[m^{-1} \sum_{k=1}^{m} z_{k} \leq T \mid H_{1}\right] \\
& \leq E_{1} \exp \left[-t\left(m^{-1} \sum_{k=1}^{m} z_{k}-T\right]\right]= \\
= & \prod_{k=1}^{m} E_{l} \exp \left[-\mathrm{tm}^{-1}\left(z_{k}-T\right)\right]
\end{aligned}
$$

for $1 \geq 0, E_{1}=$ expectation under $H_{j}$. Note that the bound ( 3 ) is less that $I$ for some $t \geq 0$, if and only if:

$$
m^{-1} \sum_{k=1}^{m} E_{o} z_{k}<T
$$

Similariy, $(t)$ is less than 1 for some $1 \geq 0$, if and only if:

$$
m^{-1} \sum_{k=1}^{m} E_{i} z_{k}>T
$$

Let. now:

$$
z_{k}=n^{-1} \log \left[g_{1 k}\left(x_{k}^{n}\right) / g_{0 k}\left(x_{k}^{n}\right)\right]
$$

be the mismatched log-likelihood function for the kth sensor. and $s=t \cdot n^{-1} \geq 0$. Straightforward calculation provides us with the following expressions:

$$
E_{0} \exp \left[m^{-1}\left(z_{k}-T\right)\right]=\int f_{0 k}\left(x_{k}^{n}\right)\left[g_{1 k}\left(x_{k}^{n}\right) / g_{0 k}\left(x_{k}^{n}\right)\right]^{s / m}
$$

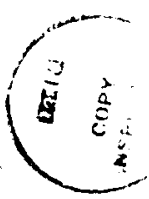

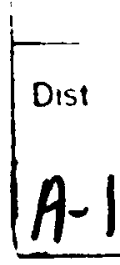

A. ii , is ior Souctal 


$$
\begin{gathered}
d x_{k}^{n} \cdot \exp (-\operatorname{snT} / m) . \\
E_{1} \exp \left[-m^{-1}\left(z_{k}-T\right)\right]=\int f_{1 k}\left(x_{k}^{n}\right)\left[g_{1 k}\left(x_{k}^{n}\right) / g_{0 k}\left(x_{k}^{n}\right)\right]^{-3 / m} \\
d x_{k}^{n} \cdot \exp (\operatorname{snT} T / m) \\
E_{0} z_{k}=n^{-1} \int f_{0 k}\left(x_{k}^{n}\right) \log \left[g_{1 k}\left(x_{k}^{n}\right) / g_{0 k}\left(x_{k}^{n}\right)\right] d x_{k}^{n} \\
E_{1} z_{k}=n^{-1} \int f_{1 k}\left(x_{k}^{n}\right) \log \left[g_{1 k}\left(x_{k}^{n}\right) / g_{0 k}\left(x_{k}^{n}\right)\right] d x_{k}^{n}
\end{gathered}
$$

Define the functionals:

$$
\begin{gathered}
G_{k}\left[f, g_{1 k}, g_{0 k}, n, s\right]^{\Delta}= \\
n^{-1} \log \int f\left(x_{k}^{n}\right)\left[g_{\mid k}\left(x_{k}^{n}\right) / g_{0 k}\left(x_{k}^{n}\right)\right]^{j / m} d x_{k}^{n}
\end{gathered}
$$

Taking logarithms of (3), (4) and using (8), (9), (12), we find the bounds:

$$
\begin{aligned}
& n^{-1} \log P_{0}(f, g, n) \leq \sum_{k=1}^{m} G_{k}\left[f_{0 k}, g_{1 k}, g_{0 k}, n, s\right]-s T . \\
& n^{-1} \log P_{1}(f, g, n) \leq \sum_{k=1}^{m} G_{k}\left[f_{1 k}, g_{1 k}, g_{0 k}, n,-s\right]+s T .
\end{aligned}
$$

We observe that if $G_{k}^{1}$ denotes the derivative of $G_{k}$ with respect to $\mathrm{s}$, then. from (10), (12), (7):

$$
\begin{aligned}
& G_{k}^{l}\left[f_{0 k}, g_{1 k}, g_{0 k}, n, 0\right]=m^{-1} E_{0} z_{k} . \\
& G_{k}^{l}\left[f_{t k}, g_{1 k}, g_{0 k}, n, 0\right]=m^{-1} E_{1} z_{k} .
\end{aligned}
$$

Hence. if (5) is sarisfied, the slope of the upper bound (13) at $s=0$ is negative, and the bound is zero at $s=0$. Similarly, if $(6)$ is satisfied. the slope of the upper bound (14) at $s=0$ is negative. and the bound is zero at $s=0$. Also. both bounds (13) and (14) are convex with respect to $s$.

The ightened Chernotf bounds (13). (14) are:

$$
\begin{aligned}
& n^{-1} \log P_{0}(f, g, n) \leq \inf _{s} \sum_{k=1}^{m} G_{k}\left[f_{0 k}, g_{\mid k}, g_{0 k}, n, s\right]-s T .(17) \\
& \left.n^{-1} \log P_{1}(f, g, n) \leq \inf _{s} \sum_{k=1}^{m} G_{k} r e \quad g_{1 k}, g_{0 k}, n,-s\right]+s T_{(18)}
\end{aligned}
$$

Suppose. now. that for the class of statistical models we consider. the limits:

$$
\lim _{n \rightarrow \infty} G_{k}\left[f_{j k}, g_{1 k}, g_{0 k}, n_{,} s\right]^{د} G_{k}\left[f_{j k}, g_{1 k}, g_{0 k}, \infty, s\right]
$$

exist, tor $k=1 \ldots . ., m, j=0.1$

Suppose. also that:

$$
\begin{aligned}
& \lim _{n \rightarrow n^{-1}} \sum_{k=1}^{m} \int f_{n_{k}}\left(x_{k}^{n}\right) \log \left[g_{1 k}\left(x_{k}^{n}\right) / g_{0 k}\left(x_{k}^{n}\right)\right] d x_{k}^{n}<T(20) \\
& \lim _{n \rightarrow-1} n^{-1} \sum_{k=1}^{m} \int f_{1 k}\left(x_{k}^{n}\right) \log \left[g_{1 k}\left(x_{k}^{n}\right) / g_{0 k}\left(x_{k}^{n}\right)\right] d x_{k}^{n}>T(21)
\end{aligned}
$$

Then, utilizing the resules of Large Deviation Theory, [8]. [9], we find:

$$
\begin{gathered}
\lim _{n \rightarrow \infty} n^{-1} \log P_{0}(\mathcal{E}, g, n)= \\
\underset{s f}{\inf } \sum_{k=1}^{m} G_{k}\left[f_{0 k}, g_{1 k}, g_{0 k}, \infty, s\right]-s T . \\
\lim _{n \rightarrow \infty} n^{-1} \log P_{1}(f, g, n)= \\
\inf _{s} \sum_{k=1}^{m} G_{k}\left[f_{l k}, g_{1 k}, g_{0 k}, \infty,-s\right]+s T
\end{gathered}
$$

It is interesting to observe that the bounds (17). (18) become the exact asymptotic error probabilities as $n \rightarrow \infty$. This is the essence of Large Deviation Theory [8]. [9].

We will now concentrate on a major class of statistical models for which the limits (19), (20), (21) exist. This is the class of Gaussian, stanonary random processes with zero means and different spectra. Consider three multuvanate Gaussian probability density functions $f\left(x^{n}\right), g\left(x^{n}\right), g_{0}\left(x^{n}\right)$ with zero means and covariance marrices $F, C_{1}, C_{0}$. We calculate the integral of eq (12):

$$
\begin{aligned}
& G\left[f, g_{1}, g_{0} n, \theta\right]=n^{-1} \log \int f\left(x^{n}\right)\left[g_{1}\left(x^{n}\right) /\left.g_{0}\left(x^{n}\right)\right|^{\theta} d x^{n}=\right. \\
& \begin{array}{c}
-\frac{1}{2}\left[n^{-1} \log \left|F_{1}^{-1}+\theta C_{1}-\theta C_{0}^{-1}\right|-n^{-1} \log \mid F_{1}^{-1}-\right. \\
\left.\quad-\theta \cdot n^{-1} \log \left|C_{1}^{-1}\right|+\theta \cdot n^{-1} \log \mid C_{0}^{-1}\right]
\end{array}
\end{aligned}
$$

Taking the limit as $n \rightarrow \infty$, and using the results of $\{10\}$. [11], we find:

$$
\begin{array}{r}
-2 G\left[f . g_{1}, g_{0}, \infty, \theta\right]=(2 \pi)^{-1} \int_{-\pi}^{\pi}\left(\log \left[f^{-1}(\lambda)+\theta c_{1}^{-i}(\lambda)-\theta c_{n}^{-1}(\lambda)\right]-\right. \\
-\log f^{-1}(\lambda)-\theta \log c_{1}^{-1}(\lambda)+\theta \log c_{0}^{-1}(\lambda) / d \lambda \quad \text { 125, }
\end{array}
$$

where, $f(\lambda), c_{1}(\lambda), c_{0}(\lambda)$ are the three spectra corresponding to $f, g_{1}, g_{0}$, and it is assumed that they are strictiy posituve for all $\lambda \varepsilon[-\pi, \pi \mid$. Using (25) for $k=1 \ldots . . . m$. and assuming that for the $k$ th sensor the orue spectra are $\left.\left\{f_{n_{k}}(\lambda), f_{1 k} \mid \lambda\right)\right\}$ under $H_{n}, H_{1}$, and the assumed spectra are $\left\{g_{0 k}(\lambda), g_{\mid k}(\lambda) \mid\right.$ under $H_{h}, H_{1}$, we can evaluate the rates in (22), (23).

The necessary and sufficient conditions in order tor $P_{n}(f, g, n)$ and $P_{1}(f, g, n)$ to converge exponenually to zero. are that the derivatuves of (22). (23) at $s=0$ are neganve. which are equivalent to (20). (21). The dervative of $G$ with respect to $\theta$ at $\theta=0$, is:

$$
\begin{gathered}
-2 G^{\prime}\left[f, g_{1}, g_{0}, \infty, \theta=0\left|=(2 \pi)^{-1} \int_{-\pi}^{\pi}\right| f(\lambda)\left[c_{1}^{-1}(\lambda)-c_{11}^{-i}(\lambda) \mid-\right.\right. \\
-\log c_{0}(\lambda) c_{1}^{-1}(\lambda) \mid d \lambda .
\end{gathered}
$$

The condition of negative slope at $s=0$ of (22) is:

$$
\begin{gathered}
(2 \pi)^{-1} \int_{-\pi}^{\pi} \sum_{k=1}^{m}\left(f_{0 k}(\lambda)\left[g_{k k}^{-1}(\lambda)-g_{0 k}^{-1}(\lambda)\right]-\right. \\
\log g_{k k}(\lambda) g_{i k}^{-1}(\lambda) \mid d \lambda+2 \mathrm{~T}>0
\end{gathered}
$$


The condition of negarive slope at $s=0$ of $(23)$ is:

$$
\begin{gathered}
(2 \pi)^{-1} \int_{-\pi}^{\pi} \sum_{k=1}^{m}\left\{f_{\mid k}(\lambda)\left\{g_{i k}^{-1}(\lambda)-g_{0 k}^{-1}(\lambda)\right\}-\right. \\
\left.\log g_{0 k}(\lambda) g_{1 k}^{-1}(\lambda)\right\} d \lambda-2 T>0 .
\end{gathered}
$$

Conditions (27), (28) guarantee exponential convergence of $\mathrm{P}_{0}, \mathrm{P}_{1}$ to zero. correspondingly. It can be shown that if (27) is not satisfied. then $P_{0}$ converges to 1 as $n \rightarrow \infty$. Similarly. if (28) is not satisfied. $P_{1}$ converges to 1 . The proof of the latter fact is not given here, but it will appear in the expanded version of the paper.

Let us define the spectral distance measure:

$$
\begin{aligned}
I(f, g)= & (2 \pi)^{-1} \int_{-\pi}^{\pi}\left\{f(\lambda) g^{-1}(\lambda)-1-\right. \\
& \left.\log f(\lambda) g^{-1}(\lambda)\right\} d \lambda
\end{aligned}
$$

Because of the identity $x-1 \geq \log x$, it is seen that $I(f, g) \geq 0$ with equality if and only if $f(\lambda)=g(\lambda)$ for almost all $\lambda \varepsilon[-\pi . \pi]$. After some algebraic manipulations, condition (27) is expressed as:

$$
\sum_{k=1}^{m} I\left(f_{0 k}, g_{i k}\right)-I\left(f_{0 k}, g_{0 k}\right)+2 T>0
$$

Similarly, condition (28) is expressed as:

$$
\sum_{k=1}^{m} I\left(f_{l k}, g_{l k}\right)-\left(f_{l k}, g_{0 k}\right)-2 T>0
$$

Combining (30) and (31), we see that the necessary and sufficient condition for exponential convergence of the error rate to zero, is the satistaction of the double inequality:

$$
\begin{aligned}
& 2^{-1} \sum_{k=1}^{m} I\left(f_{1 k}, g_{1 k}\right)-I\left(f_{1 k}, g_{0 k}\right)>T> \\
& >2^{-1} \sum_{k=1}^{m} I\left(f_{0 k}, g_{0 k}\right)-I\left(f_{0 k}, g_{1 k}\right) .
\end{aligned}
$$

As long as the leftmost side of (32) is larger than the rightmost side. we can always pick a threshold $T$ between those rwo numbers to achieve asymptotic convergence of the error rates to zero. For the special case of matched staustics. we have $i_{i k}=g_{1 k}, f_{0 k}=g_{0 k}$, and the condition (32) becomes:

$$
2^{-1} \sum_{k=1}^{m} I\left(f_{1 k}, f_{0 k}\right)>T>-2^{-1} \sum_{k=1}^{m} I\left(f_{0 k}, f_{l k}\right)
$$

We note that (33) can always be satisfied for some $T$, because the leftmost side is nonnegative. and the rightmost side nonpositive. Thus. in the "matched" case. exponential convergence hinges only upon the choice of $T$.

To evaluate the actual rates of convergence. we need to use expression (25) for $k=1$.....m into (22). (23) and minimize with respect to s.

\section{CONCLUSIONS}

We have obtained the rates of convergence of error probabilities in multisensor detection for a binary hypothesis, and we determined the conditions of exponential convergence in the presence of mismatch. The conditions are necessary and sufficient.

\section{REFERENCES}

[1] Tenney, R. R. and Sandell, N. R. (1981) "Derection with distributed sensors," IEEE Transactions on AES, July 1981, pp. 501-510.

[2] Teneketzis. D. (1982) "The decentralized quickest detection problem." Proceedings of the 21st [EEE Conf. on Decision and Control.

(3) Srinivasan, R (1986), "Distributed Padar Detection Theory," IEE Proceedings. vol 133, ptF. Feb 1986. pp. 55-60.

[4] Chair, Z and Varshney, P. (1987) "Neymann-Pearson Hypothesis Testing in Distributed Networks." 1987 Conf. on Decision and Control.

[5] Reibman. A. R. and Nolte, L. W. (1987) "Optimal Detection and Performance of Distributed Sensor Systems," IEEE Trans. on AES, vol 23. no 1. pp. 24-30.

[6] Viswanathan, R. Thomopoulos, S.C.A.. Tumuiun. R., "Spatal Serial Distributed Decision Fuston. 1987 IEEE Conf. on Decision and Control.

(7) Reibman. A. R. and Nolte. L. W., "Design and Performance Comparison of Distributed Detecton Vietworks." IEEE Trans. on AES, Vol 23. no 6, Nov 1987, pp 789-797.

[8] Chemoff. H. (1952), "A measure of asymptotic e:ficiency for tests of a hypothesis based on the sum of observations." Ann. of Math. Stat. vol 23. pp. 493. 507, 1952.

[9] Ellis, R. S. (1984), "Large Deviations for a General Class of Random Vectors." Annais of Prob., vol i2. pp 1-12, 1984.

[10] Kazakos, D. and Papantoni-Kazakos. P. (1980). "Spectral Distance Measures Between Gausstan Processes." IEEE Trans. on Aut. Control, vol AC.25. no 5. October 1980. pp 950-959.

[11] Kazakos, D. (1982), "Statistical Discrimination Using Inaccurate Models," IEEE Trans. on Intor. Theory, Sept 1982. vol IT-28. 


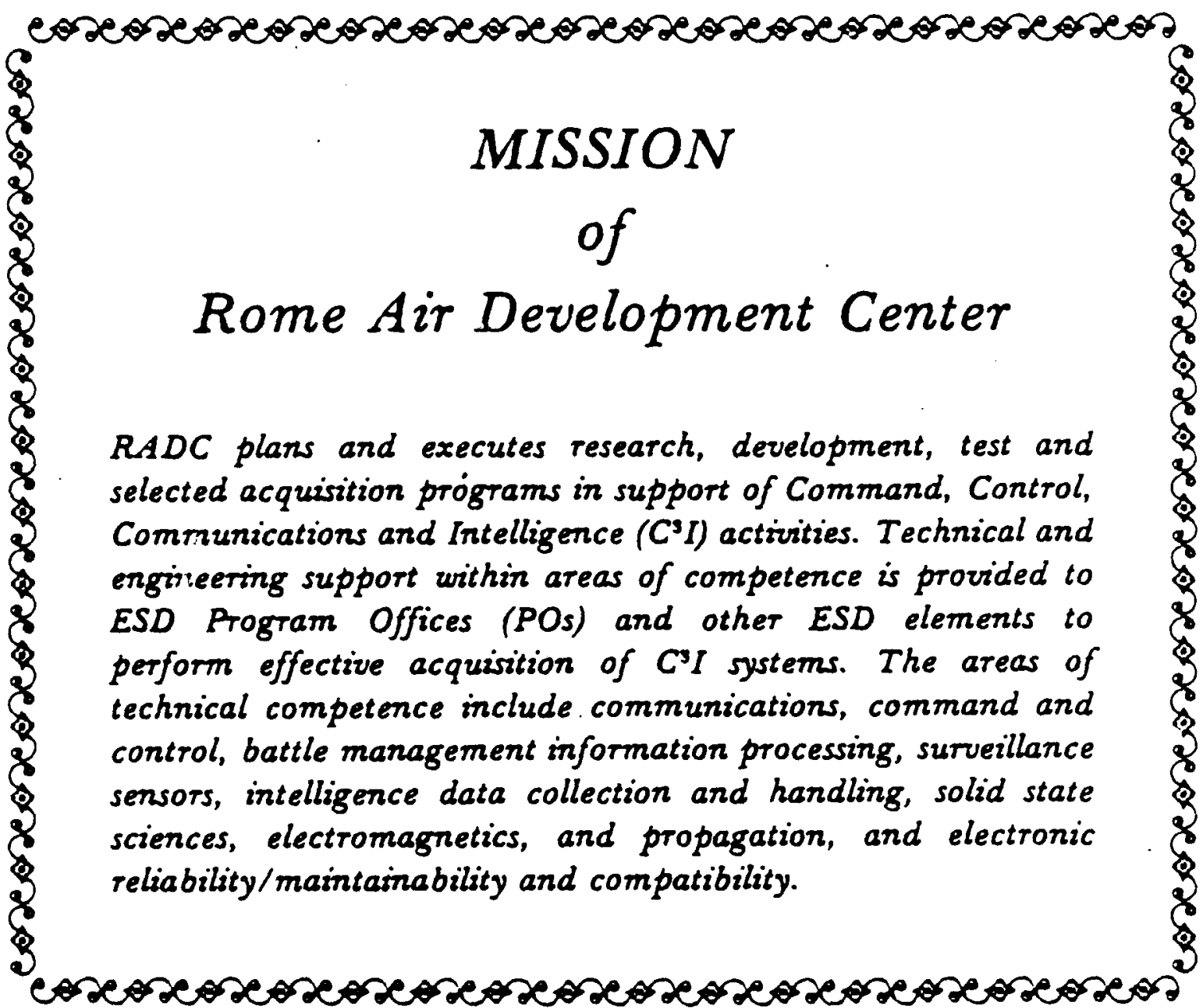

